\section{Research Square}

Preprints are preliminary reports that have not undergone peer review.

They should not be considered conclusive, used to inform clinical practice, or referenced by the media as validated information.

\title{
Development of a remote online collaborative medical school pathology curriculum with clinical correlations, across several international sites, through the covid-19 pandemic
}

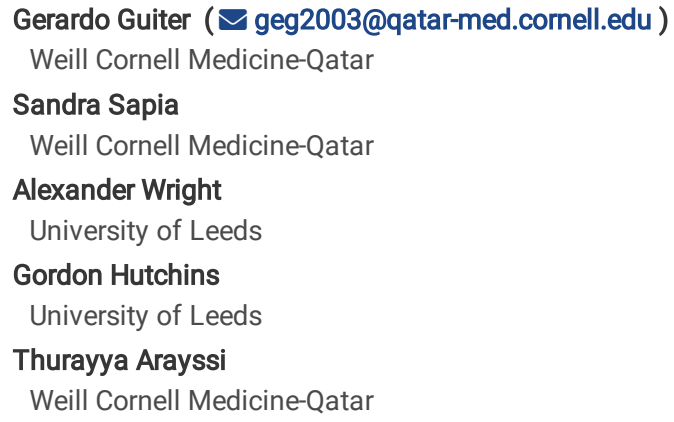




\section{Abstract}

Introduction: Due to the Covid-19 social distancing restrictions, in March 2020, Weill Cornell Medicine-Qatar decided to replace students' clinical instruction with novel online electives. Hence, we implemented an innovative online and remote pathology curriculum, anchored on virtual microscopy and Zoom videoconferencing: ideal tools to support online teaching.

Objective: To assess a new curriculum implementation at Weill Cornell Medicine-Qatar.

Materials and Methods: This for-credit, 2-week elective included 6 synchronous Zoom sessions where complex clinicopathological cases were discussed in small groups. We used open access digital microscopy slides from the University of Leeds' Virtual Pathology Library

(http://www.virtualpathology.leeds.ac.uk/slides/library/). Students independently prepared for these sessions by reviewing cases, slides, readings, and questions in advance (asynchronous self-directed learning anchored on a flipped classroom model), and wrote a final review of a case. An assessment and feedback were given to each student.

Results: Four elective iterations were offered to a total of 29 students, with learners and faculty spread over 4 countries. During the Zoom sessions, students controlled the digital slides and offered their own diagnoses, followed by group discussions to strengthen autonomy and confidence. We surveyed learners about the elective's performance (program evaluation). Students conveyed high levels of satisfaction about the elective's overall quality, their pathology learning and online interactions, with minimal challenges related to the remote nature of the course.

Discussion and conclusions: Technological innovations mitigate sudden disruptions in medical education. A remote curriculum allows instruction at any distance, at any time, from anywhere, enhancing educational exchanges, flexibility and globalization in medical education.

\section{Introduction}

Weill Cornell Medicine-Qatar (WCM-Q) is an international site of Weill Cornell Medicine. Established in 2001 as a partnership between Cornell University and Qatar Foundation, WCM-Q is part of Cornell University in Ithaca, New York, and shares the tripartite mission of Weill Cornell Medicine-New York (WCM-NY): a dedication to excellence in education, patient care, and research. It offers an integrated program of pre-medical and medical studies leading to the Cornell University MD degree.

WCM-Q medical school curriculum is guided by key principles of enhancing the integration between the foundational sciences and clinical learning, and introducing students to patient care from the first day of their studies. The curriculum is divided into 3 phases. During Phase 1 , which extends over 18 months, students complete their foundational sciences courses. Phase 2 is dedicated to 12 months of clinical clerkships at primary and tertiary care academic centers in Doha, Qatar. Phase 3 focuses on advanced clinical experiences and the completion of a scholarly capstone project.

Due to the global Covid-19 pandemic, in March 2020, WCM-Q suspended all clinical instruction in a move aligned with the Association of American Medical Colleges (AAMC) and the Liaison Committee on Medical Education (LCME) recommendations [1-3] and similar decisions by other educational institutions in North America, Asia, and Europe [4-7]. This approach to protecting students was sensible particularly in light of past experiences, such as when 16 medical students exposed to severe acute respiratory syndrome (SARS) patients in a hospital during the Hong Kong 2003 outbreak unfortunately contracted the disease [8].

As a result, our institution faced a sudden challenge: to adapt to the new situation and enable our students to progress in the curriculum safely. Consequently, WCM-Q decided to replace our learners' clinical work with a portfolio of novel electives to be delivered entirely online starting in mid-April 2020 , covering various fields of expertise. The strategy included offering 2-or 4-week electives to provide students with enhanced flexibility if they needed to return to the clinical space on short notice.

At that challenging time, the authors of this project believed that pathology teaching offered a superb solution for our institutional needs, based on the use of virtual microscopy and digital slides. These are ideally suited for delivering an undergraduate pathology curriculum [9], and many institutions in North America and Europe have already switched to this approach [10-12]. Indeed, WCM-Q has been using virtual slides for pathology and histology instruction throughout all basic science courses since 2005, and the American Board of Pathology certification exam began using virtual microscopy in 2003 [13]. Large academic medical centers have ample experience with virtual microscopy and employing digital slides for undergraduate teaching [14] and clinical diagnostic purposes [15], and most recently, during the Covid-19 pandemic [16, 17]. In the US, a corresponding relaxation of government regulations has allowed pathologists to review and report pathology specimens from remote, non-Clinical Laboratory Improvement Amendments (CLIA) certified facilities [17], expanding the use of digital slides with remote reviews.

However, virtual microscopy and digital slides in medical school education have traditionally accompanied face-to-face "in-situ" interactions, with very few remote applications and mostly targeting post-graduate training [18, 19]. To communicate with our learners within the social distancing restrictions imposed by the Covid-19 pandemic, our project sought to develop and implement an undergraduate innovative pathology collaborative learning experience. Students

Page $2 / 9$ 
and instructors would connect remotely and share--synchronously and asynchronously--virtual microscopy, digital slides, and other images, as well as discussions, questions, and answers through the Zoom videoconferencing system, currently available at WCM-Q as an institutional subscription. We targeted WCM-Q medical students that had already completed their foundational sciences courses.

\section{Materials And Methods}

\section{Design of the Elective}

We designed a 2-week online elective with the overarching goal that learners, by focusing on the study of several malignant tumors and their precursor lesions, would acquire an enhanced knowledge of malignant diseases and therefore the basis for robust understandings of oncologic pathology and oncology. The elective was available for any student who had successfully completed our foundational sciences curriculum. Students passing the elective would gain appropriate credit as determined by institutional policy.

The elective included a total of 6 interactive, synchronous live sessions using Zoom videoconferencing ( 3 sessions per week). We decided to accept a maximum of 10 students per elective iteration, believing that an instructional approach based on small groups best suited our program, supporting increased student participation and positive learning outcomes.

Two WCM-Q and Department of Pathology and Laboratory Medicine WCM-NY faculty members developed 9 clinicopathological cases with corresponding virtual slides from the University of Leeds Virtual Pathology Slide Library (http://www.virtualpathology.leeds.ac.uk/slides/library/). This open access collection of slides is digitized and curated by trained pathologists and stored with anonymized clinical information, used for teaching and training. Slides can be viewed on standard computer screens and mobile devices and are globally accessible. All cases included an initial clinical vignette describing a patient presentation and diagnostic imaging and laboratory results, followed by biopsy and/or surgical excision histopathological findings. The cases also presented illustrations of gross specimens, immunohistochemical images, and for some, descriptions and results of advanced molecular studies. The cases focused on uterine cervical, colonic, pulmonary, and mammary oncologic pathology, and included a list of differential diagnoses, with the University of Leeds Slide Library virtual slides illustrating alternative but meaningful and related pathological entities.

As our foundational sciences curriculum at WCM-Q includes general and systemic pathology, our students had already acquired a basic knowledge of pathology. We accordingly created cases that were somewhat more complex than those our learners had already studied, so they would continue incorporating new information and constructing new understandings in pathology throughout this elective.

We included in all cases 2 types of questions aimed at topics addressed during the sessions: first order basic queries and more complex United Stated Medical Licensing Examination (USMLE) type items. Students were expected to answer these questions to the best of their knowledge drawn from readings and general preparation. We included the USMLE items with the following 3 objectives: first and most importantly, to ensure students had learned their materials and increased their knowledge; second, to strengthen their self-directed learning (SDL) and subsequent self-assessment skills; and third, to contribute to their preparation for the USMLE Step 1, an exam taken by most of our learners prior to graduation.

We decided to interactively teach and connect with the students via the Zoom videoconferencing platform. Zoom allows for screen sharing and remote control by any participant--a significant plus, we believed, that could enhance learner autonomy and self-discovery skills by granting students full control to point out specific areas while describing pathology or radiology images. In addition, we also chose to make use of the built-in Zoom polling as another way to increase student contributions during case discussions.

As we planned the elective, we decided that students would be expected to independently prepare for these Zoom sessions by reviewing the cases and digital slides, completing assigned readings, and answering all questions in advance (asynchronous SDL). This independent preparation was factored into the students' assessment. Therefore, in order to support our students' success, the program comprised two components of approximately equal importance: a preparatory asynchronous SDL step followed by synchronous interactive sessions with live Zoom discussions.

The elective was hosted on the WCM-Q Canvas Learning Management System (LMS). Each iteration included a unique centralized site on Canvas, allowing for faculty and learner interaction, student submissions, access to teaching files, images, and other documents, and assessment of students after completion of the elective. 
Students were also tasked with writing a final 500-word summary review of a case discussed during the elective, addressing all significant pathological and related clinical issues. The review was open-ended, and no written rubric was provided to the students; instead, they were given verbal instructions at the beginning and end of the elective.

A final written assessment of each student's performance included several items graded on a 5-point Likert type rating scale, including formative and summative comments. In addition, the faculty provided oral formative feedback to the students through one-on-one Zoom meetings. The final course grade was assigned as Pass/Fail.

This project was submitted to the WCM-Q Institutional Review Board (IRB) and determined IRB exempt.

\section{Results}

\section{Implementation of the Elective}

Four elective iterations were offered to a total of 29 students between April 19 and June 25 . The first iteration included 10 students, the second included 5 students, and the third and fourth iterations included 7 students each. Learners and faculty were spread over 4 different countries (Qatar, Saudi Arabia, Peru and Argentina).

The 2 participating WCM-Q/WCM-NY faculty facilitated the Zoom instruction. Following the program design, we met with students 3 times per week for sessions lasting 90 to 120 minutes. While discussing cases, the students had many opportunities to take control of the virtual slides, moving the images and zooming in and out at their discretion, and selecting areas of interest to share with the group. They also described their initial preliminary pathological diagnoses based on their evaluation of the virtual slides. In so doing, the learners "acted" as pathologists in control of their cases, similar to what happens at pathology departments during consensus meetings where difficult cases are shown to colleagues. We did not experience any difficulties with the Zoom platform, which worked very well during all sessions, and encountered no "Zoombombing" [19].

\section{Assessment of the Elective and Students' Feedback}

We surveyed our students to acquire some preliminary impressions and data about the elective's performance and functioning (program assessment), and to make any necessary changes. We aimed to evaluate the elective's overall quality and the quality of student and faculty online interactions, our students' online and remote learning of pathology, any challenges associated with their work and communications, and the most/least effective aspects of their remote learning experiences. We acknowledged that this appraisal would only be based on our learners' input, assessment, and feedback.

A 2-part, anonymous survey, prepared by the WCM-Q Office of Educational Development (OED) for all virtual elective courses, was distributed to the 29 students who had completed our pathology elective, and 23 (79.31\%) responded. Table 1 and Table 2 summarize the survey results.

We additionally asked the students if they had or had not experienced any challenges due to their remote participation in this course. Only 1 out of 23 (4.35\%) respondents answered that they had, noting "some lag in the communications."

Finally, we asked the students to describe the most and the least effective aspects of their remote learning experiences. Virtual pathology slides, ability to communicate and engage more in the activities through online learning rather than when it is face-to-face,

easier communication with the professors and more relaxed environments at home,

solving the cases prior to the sessions and inclusion of USMLE questions were reported as highly effective. No comments were received on the least effective aspects of the course.

During the four iterations of the course, we encountered loss of Internet during 1 of 24 sessions (4.16\%), which affected the connectivity of a few students. This session was re-scheduled for the next day, resolving the problem. We also encountered loss of the virtual microscopy website connectivity during 2 of 24 sessions (8.33\%). This issue, though not ideal, was solved by sharing static images (JPGs) instead, prepared in advance from the same corresponding digital slides. 
While these technical troubles were rare, they underscored the critical importance of developing Internet and server back-up systems for institutions that offer online courses. In addition, although during our elective we used standard home-type Internet, networks that offer adequate bandwidth are crucial. In summary, efforts must be made to mitigate technical risks that can diminish student satisfaction and participation. Every student passed the elective.

\section{Discussion}

This project involved the development and implementation of a novel undergraduate pathology curriculum at WCM-Q, built on the use of virtual microscopy, with learners and faculty interacting remotely by Zoom videoconferencing--a notably original approach in pathology teaching in medical school education.

Fortunately, we were able to design and implement this elective swiftly, as our appraisal of existing infrastructure confirmed that all the resources needed were already available. These included Zoom videoconferencing, Canvas, Information Technology assistance, secretarial support, OED collaboration, and the University of Leeds virtual slides. In addition, we obtained crucial WCM-Q leadership support, as evidenced by their quick approval of the elective for credit recognition and by ensuring that all the resources were readily accessible.

During our needs assessment, we also considered the characteristics of our targeted learners. First, they are tech savvy, critical in this elective, which is heavily reliant on technology. Second, they are familiar with flipped classrooms and therefore were well prepared for our elective entailing many aspects of the flipped classroom model. Third, our students have strong pathology foundations and are familiar with clinicopathological cases and virtual slides, since pathology teaching during the foundational courses at WCM-Q is grounded on these tools. Finally, we did not encounter any barriers to this elective. All of these factors mentioned above are critical for the implementation and success of a new medical school curriculum.

The program was very well received by the students. Based on their written and oral feedback, the remote nature of the course presented minimal challenges. Learners reported high satisfaction with the opportunities to interact online with peers and faculty, and with their learning of pathology and related topics. Indeed, some students mentioned they preferred this mode of teaching over face-to-face instruction. Reasons for this predilection included enhanced time management, no time lost in transportation, and a lack of distraction by peers (as sometimes happens during instruction at the college). In addition, we as faculty did not encounter any difficulties with the remote delivery of our curriculum.

Our program was a response to the sudden need to interact with students at a distance. We switched from a traditional teaching paradigm of face-to-face interactions with our learners to a new ideal approach of $100 \%$ remote exchanges. This experience parallels similar needs and responses in the clinical arena. For example, in the US as a result of this pandemic, hospital pathologists have been allowed to temporarily switch reporting of cases to remote locations, following guidance by the College of American Pathologists (CAP) and the Centers for Medicare and Medicaid Services (CMS) [20-22]. In fact, as per CMS guidance, a temporary testing site may even include the pathologist's home.

We were also interested in whether students carried on developing their SDL and self-discovery skills through this program. They were accordingly provided with cases and access to virtual slides, readings, and other activities 72 hours in advance of Zoom meetings. During their preparatory time, the learners reviewed these items on their own and were expected to create differential diagnoses, eventually formulating their own specific cancer diagnosis. Thus, this preparatory time was a great opportunity for SDL and self-discovery in line with LCME standards for accreditation of medical education programs [23] and World Federation for Medical Education (WFME) basic medical education global standards for quality improvement [24].

During the Zoom meetings, students were first asked to share with the group the diagnoses they had generated during their preparatory time. This was followed by a group discussion and a final consensus diagnosis under faculty guidance. Students controlled the virtual slides during the discussion, selecting and showing to others their fields of interest and important tumor features to support their diagnoses. This learning strategy can strengthen student autonomy and confidence, and eventually, self-efficacy and motivation for learning. Faculty also offered formative feedback and reinforcements. In addition, sharing images, ideas, and questions and answers live through Zoom enhanced collaborative work and learning. In fact, sometimes students offered different perspectives on a difficult case, encouraging rich discussions during our meetings.

\section{Conclusions And Future Directions}

Our experience demonstrates how enhanced flexibility and technological innovations can now effectively relieve and mitigate sudden disruptions in undergraduate medical education in the foundational sciences due to a viral pandemic, or for any other cause.

This is not the first time medical education has been affected by an infectious outbreak. The 2003 Hong Kong SARS and 2015 South Korea Middle East Respiratory Syndrome (MERS) outbreaks mandated barring students from patient contact, cancelling clerkships, and closing medical schools [25-27]. Luckily, although the COVID-19 pandemic has harshly impacted education at many levels, we now have instructional tools previously unavailable. Still, it is imperative

Page 5/9 
to learn from recent events, since the possibility exists of future analogous challenges. The current pandemic has encouraged instructional innovations that must be included in our medical education armamentarium.

A benefit of this remote curriculum is that it allows instruction at any distance, for any reason, at any time, and from anywhere. This supports flexibility and globalization in medical education. As virtual teaching becomes more generalized, it will allow students to interact with peers and faculty at other institutions worldwide, supporting and increasing educational exchanges.

Remote education, however, cannot replace face-to face instruction. We favor a blended system, including distanced and face-to-face teaching. Moreover, some key aspects of medical education (e.g., bedside teaching), can only occur in the context of direct contact with patients.

It is also important that policymakers and governing bodies ensure that medical schools have all the necessary resources to face the next "emergency" in undergraduate medical education. This includes technology and faculty development. Similarly, it would be useful for appropriate national (e.g., LCME in the US) and supranational (e.g., WFME) organizations to establish some common rules, standards, and guidelines for medical education at a distance so that high-quality remote instruction is assured and maintained.

Lastly, our work should be replicated by others and evaluated accordingly. Future research should look at hard evidence of learning outcomes associated with remote online instruction.

\section{Tables}

\begin{tabular}{|c|c|c|}
\hline & Mean $^{a}$ & Standard Deviation \\
\hline Elective's overall quality & 4.9 & 0.34 \\
\hline Clarity of elective's goals and objectives & 4.9 & 0.34 \\
\hline Elective's organization (syllabus, schedule, learning arrangements, support mechanisms) & 4.8 & 0.41 \\
\hline Elective's contribution to the students' learning and understanding of oncologic pathology & 4.8 & 0.51 \\
\hline
\end{tabular}

Table 2: Survey of students about the elective's performance (program assessment)-Part B

\begin{tabular}{|c|c|c|}
\hline & Mean $^{\mathrm{a}}$ & $\begin{array}{l}\text { Standard } \\
\text { Deviation }\end{array}$ \\
\hline After completing this elective, my knowledge of some complex pathological entities and diseases has improved & 4.6 & 0.49 \\
\hline After completing this elective, my recognition of the critical importance of robust clinicopathological correlations has improved & 4.6 & 0.49 \\
\hline $\begin{array}{l}\text { After completing this elective, my comprehension of the rationale supporting targeted therapies and preventive approaches in } \\
\text { malignancies has improved }\end{array}$ & 4.4 & 0.65 \\
\hline The elective's Zoom sessions encouraged interactions between students & 4.8 & 0.39 \\
\hline The elective's Zoom sessions encouraged interactions between faculty and students & 4.7 & 0.44 \\
\hline
\end{tabular}

\section{Declarations}

- Funding: No funding was received for conducting this study

- Conflicts of interest/Competing interests: None. The authors have no relevant financial or non-financial interests to disclose

- Ethics approval: This project was submitted to the Weill Cornell Medicine-Qatar Institutional Review Board (IRB) and determined IRB exempt

- Consent to participate: Not applicable

- Consent for publication: The authors affirm that Weill Cornell Medicine-Qatar students provided informed consent for publication of their photographs in Figure 1 
- Availability of data and material: all data and materials support the published claims and comply with field standards. All data generated or analysed during this study are included in this article

- Code availability: Not applicable

\section{References}

1. Whelan A, Prescott J, Young G, Catanese VM. Guidance on Medical Students' Clinical Participation: Effective Immediately. March 17, 2020. https://lcme.org/wp-content/uploads/filebase/March-17-2020-Guidance-on-Mediical-Students-Clinical-Participation.pdf. Accessed 1 August 2020.

2. Whelan A, Prescott J, Young G, Catanese VM, McKinney R. Interim Guidance on Medical Students' Participation in Direct Patient Contact Activities: Principles and Guidelines. March 30, 2020. https://lcme.org/wp-content/uploads/filebase/March-30-2020-Interim-Guidance-on-Medical-StudentsParticipation-in-Direct-Patient-Contact-Activities.pdf. Accessed 1 August 2020.

3. Whelan A, Prescott J, Young G, Catanese VM, McKinney R. Guidance on Medical Students' Participation in Direct Patient Contact Activities. April 14, 2020. https://lcme.org/wp-content/uploads/filebase/April-14-2020-Guidance-on-Medical-Students-Participation-in-Direct-Patient-Contact-Activities.pdf. Accessed 1 August 2020

4. Dedeilia A, Sotiropoulos MG, Hanrahan JG, Janga D, Dedeilias P, Sideris M. Medical and surgical education challenges and innovations in the COVID-19 era: a systematic review. In Vivo. 2020; https://doi:10.21873/invivo.11950

5. Liang ZC, Ooi SBS, Wang W. Pandemics and their impact on medical training: lessons from Singapore [published online April 17, 2020]. Acad Med. 2020; https://doi:10.1097/ACM.0000000000003441

6. Yurtass IS, Aktar I, Bardak M, Goeschl S. European Medical Students' Association. Institutional Report for COVID-19 Impact on Medical Education. https://drive.google.com/file/d/1NCD0F8CLixFR_QPCFzYSdGTf5dAvBbVr/view. Accessed 1 August 2020.

7. Esposito S, Principi N. School closure during the coronavirus disease 2019 (COVID-19) pandemic: an effective intervention at the global level? JAMA Pediatr.2020; https://doi:10.1001/jamapediatrics.2020.1892

8. Lee N, Hui D, Wu A, et al. A major outbreak of severe acute respiratory syndrome in Hong Kong. N Engl J Med. 2003; https://doi:10.1056/NEJMoa030685

9. Vainer B, Mortensen NW, Poulsen SS, et al. Turning microscopy in the medical curriculum digital: experiences from the faculty of Health and Medical Sciences at University of Copenhagen. J Pathol Inform. 2017;8:11.

10. Shahriari N, Grant-Kels J, Murphy MJ. Dermatopathology education in the era of modern technology. J Cutan Pathol. 2017; https://doi.org/10.1111/cup.12980

11. Kuo KH, Leo JM. Optical versus virtual microscope for medical education: a systematic review. Anat Sci Educ. 2019; https://doi:10.1002/ase.1844

12. Lee BC, Hsieh ST, Chang YL, et al. A web-based virtual microscopy platform for improving academic performance in histology and pathology laboratory courses: a pilot study [published online December 27, 2019]. Anat Sci Educ. 2019; https://doi.org/10.1002/ase.1940 
13. Lee S-H. Virtual microscopy: applications to hematology education and training. Hematology. 2005; https://doi.org/10.1080/10245330512331390249

14. Randell R, Hutchins G, Sandars J, et al. Using a high-resolution wall-sized virtual microscope to teach undergraduate medical students. In: $\mathrm{CHI}$ ' 12 Extended Abstracts on Human Factors in Computing Systems (CHI EA '12). Association for Computing Machinery; 2012; https://doi.org/10.1145/2212776.2223815

15. Williams BJ, Hanby A, Millican-Slater R, Nijhawan A, Verghese E, Treanor D. Digital pathology for the primary diagnosis of breast histopathological specimens: an innovative validation and concordance study on digital pathology validation and training. Histopathology. 2018; $72(4): 662-671$.

16. Williams BJ, Brettle D, Aslam M, et al. Guidance for remote reporting of digital pathology slides during periods of exceptional service pressure: an emergency response from the UK royal college of pathologists. J Pathol Inform. 2020;11:12.

17. Hanna MG, Reuter VE, Ardon O, et al. Validation of a digital pathology system including remote review during the COVID-19 pandemic [published online June 22, 2020]. Mod Pathol. 2020; https://doi:10.1038/s41379-020-0601-5

18. Kim RH, Brinster NK, Meehan SA. Dermatopathology education during the COVID-19 pandemic: virtual simulation of the multi-headed microscope [published online June 1, 2020]. J Am Acad Dermatol. 2020; https://doi.org/10.1016/j.jaad.2020.05.12

19. Mukhopadhyay S, Booth AL, Calkins SM, et al. Leveraging technology for remote learning in the era of COVID-19 and social distancing: tips and resources for pathology educators and trainees [published online May 4, 2020]. Arch Pathol Lab Med. 2020; https://doi.org/10.5858/arpa.2020-0201-ED

20. Digital and Computational Pathology Committee and the Informatics Committee. Remote sign-out of cases with digital pathology FAQs. College of American Pathologists website.https://www.cap.org/covid-19/remote-sign-out-faqs. Cited by: Hanna MG, Reuter VE, Ardon O, et al. Validation of a digital pathology system including remote review during the COVID-19 pandemic [published online June 22, 2020]. Mod Pathol. 2020; https://doi.org/10.1038/s41379-020-0601-5

21. College of American Pathologists. CAP secures remote work waiver for pathologists. College of American Pathologists website. March $26,2020$. https://www.cap.org/advocacy/latest-news-and-practice-data/march-26-2020. Accessed 1 August 2020.

22. Center for Clinical Standards and Quality/Survey \& Certification Group. Clinical laboratory improvement amendments (CLIA) laboratory guidance during COVID-19 public health emergency. Centers for Medicate and Medicaid Services website. March 26, 2020. https://www.cms.gov/medicareproviderenrollment-and-certificationsurveycertificationgeninfopolicy-and-memos-states-and/clinical-laboratory-improvement-amendments-clia-laboratoryguidance-during-covid-19-public-health. Cited by: Hanna MG, Reuter VE, Ardon O, et al. Validation of a digital pathology system including remote review during the COVID-19 pandemic [published online June 22, 2020]. Mod Pathol. 2020; https://doi.org/10.1038/s41379-020-0601-5

23. Functions and Structure of a Medical School. Standards for Accreditation of Medical Education Programs Leading to the MD Degree. LCME. March 2020. https://lcme.org/publications/ Accessed 1 August 2020.

24. World Federation For Medical Education. Basic Medical Education WFME Global Standards For Quality Improvement. The 2015 Revision. WFME. https://wfme.org/download/wfme-global-standards-for-quality-improvement-bme/? wpdmdl=831\&refresh=5f58d5fc3ae221599657468\%22\%3EDownload\%3C/a\%3E\%20\%20\%20\%20\%20\%20\%20\%20\%20\%20\%20\%20\%3C/div\%3E\%20\%20 Accessed 1 August 2020

25. McGugan S. Medical school on bypass during the SARS outbreak. Clin Invest Med. 2003;26:106-107. Cited by: Lim EC, Oh VM, Koh DR, Seet RC. The challenges of "continuing medical education" in a pandemic era. Ann Acad Med Singapore. 2009;38(8):724-726. 
26. Patil NG, Chan Y, Yan H. SARS and its effect on medical education in Hong Kong. Med Educ. 2003; https://doi.org/10.1046/j.1365-2923.2003.01723.x Cited by: Lim EC, Oh VM, Koh DR, Seet RC. The challenges of "continuing medical education" in a pandemic era. Ann Acad Med Singapore. 2009;38(8):724-726.

27. Park SW, Jang HW, Choe YH, et al. Avoiding student infection during a Middle East respiratory syndrome (MERS) outbreak: a single medical school experience. Korean J Med Educ. 2016; https://doi.org/10.3946/kjme.2016.30

\section{Figures}

\section{Virtual Pathology at the University of Leeds}

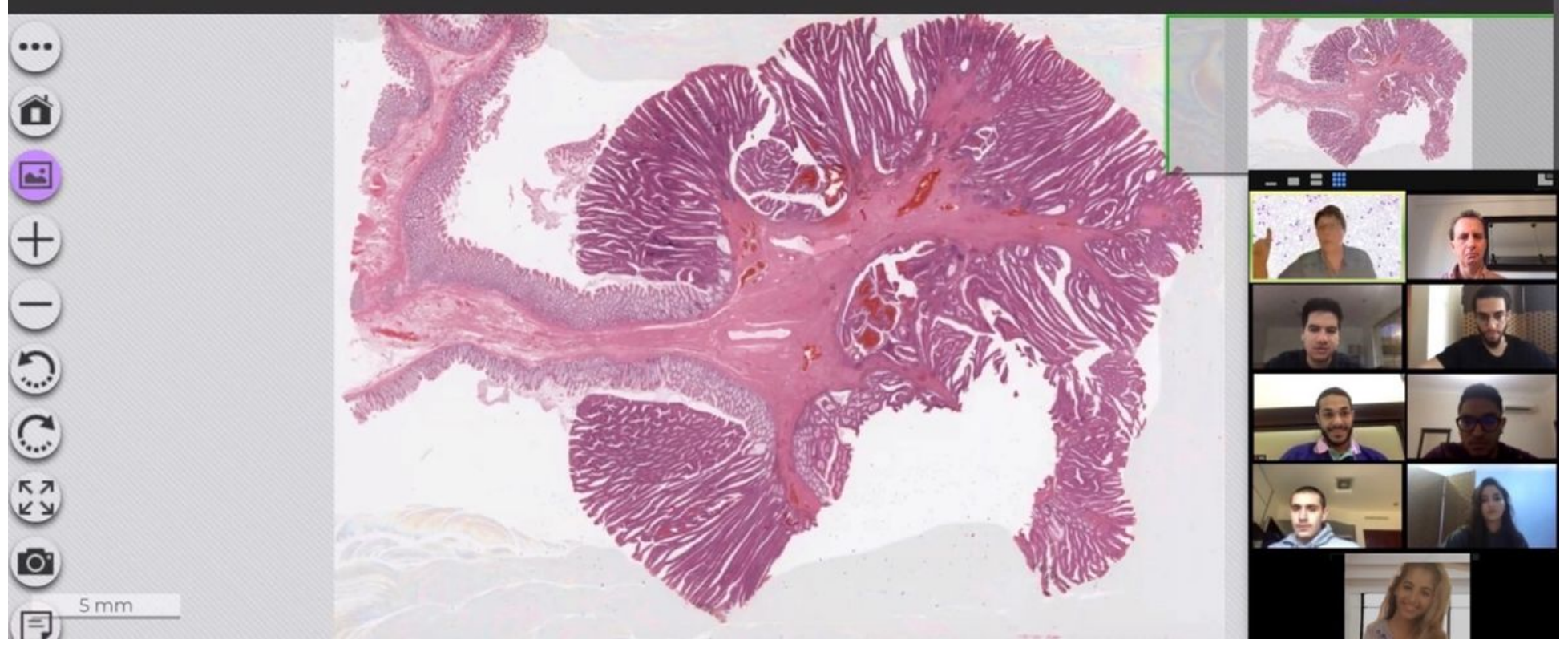

\section{Figure 1}

WCM-Q faculty and students discussing a virtual microscopy pathology slide via Zoom videoconferencing 